\section{Beat Hanselmann}

\section{Materia Medica und Repertorien}

Die genaue Anleitung zur Ausübung der Homöopathie finden wir im Organon [1]. Seit seinem ersten Erscheinen 1810 ist es Lehrbuch und Referenz für alle. Später kamen die zwei wichtigsten Hilfsmittel oder Werkzeuge hinzu, zuerst die Materia Medica, danach die Repertorien.

Die Arzneimittelprüfung am Gesunden enthüllt uns die Heilkräfte der einzelnen Arzneien. Von den Prüfungsprotokollen und der daraus zusammengestellten Arzneimittellehre «sey alles Vermuthete, blo $\beta \mathrm{Be}$ hauptete, oder gar Erdichtete gänzlich ausgeschlossen; es sey alles reine Sprache der sorgfältig und redlich befragten Natur» [1; \$144]. Mit der ab 1811 erscheinenden «Reinen Arzneimittellehre» [2] und den ab 1828 erscheinenden «Chronischen Krankheiten» [3] zeigte Hahnemann seinen Kollegen, wie eine solche Materia Medica pura aussehen kann: Die Symptome werden von Kopf zu Fuss in einem einheitlichen Schema gegliedert und nummeriert, Heilwirkungen und unsichere Symptome gekennzeichnet sowie Prüfer oder Quelle angegeben. Mögliche klinische Indikationen sind lediglich im Vorwort kursorisch aufgeführt. In späteren Arzneimittellehren präzisierten die Autoren die reinen Prüfungssymptome und ergänzten sie mit klinischen Erfahrungen, die aus der Anwendung der Arznei im Krankheitsfall gewonnen wurden.

Je umfangreicher die Materia Medica wurde, desto schwieriger war es, sich an Symptome zu erinnern oder sie einer bestimmten Arznei zuzuordnen. Es entstanden die Repertorien, eine Art «Inhaltsverzeichnis» zur

\title{
Das Symptomen-Lexikon
}

Materia Medica. Arzneimittellehre und Repertorium stehen in einem Wechselverhältnis: Die Materia Medica speist das Repertorium, das Repertorium verweist auf die Materia Medica. Heute werden Fälle zum Teil allein mit dem Repertorium gelöst. Dies erachteten die «alten» Homöopathen als keine gute Idee und war nicht wünschenswert. Ein Repertorium «kann nichts Anderes als Winke auf die vorhandnen Arznei-Prüfungen enthalten» [4; S. 102].

Im Umgang mit diesen beiden grundlegenden Hilfsmitteln bzw. Werkzeugen offenbaren sich im Praxisalltag aber auch ihre Schwachstellen. In der Materia Medica wird jedes Symptom nur einmal aufgeführt, angeordnet nach seiner Lokalisation (Kopf-zu-Fuss-Schema). Nur selten und ansatzweise sind Verweise auf ähnliche Symptome innerhalb derselben oder einer anderen Arznei angegeben (vergleiche z.B. Hahnemanns Anmerkungen bei China [2; Band 3]). Beim Repertorium sind zwar möglichst alle Vergleichsmittel aufgelistet, es fehlen aber die genaueren oder differenzierenden Angaben.

\section{Symptomen-Lexikon und Kollektaneen}

Seit einigen Jahren gibt es ein neues Hilfsmittel bzw. Werkzeug, das eine Stellung zwischen Materia Medica und Repertorium einnimmt: das Symptomen-Lexikon. Der Wunsch danach bestand seit den Anfängen der Homöopathie; schon Hahnemann hatte die Idee dazu. Es gab zwar ein paar vereinzelte Ansätze zu einem solchen Werk, etwa Gottlieb Heinrich Georg Jahrs «Repertorium» zu seinem
«Symptomen-Kodex» von 1848 [5] oder die «Systematischen Darstellungen» von Georg Adolph Weber aus dem Jahr 1830 [6] und von Ernst Theodor Ferdinand Rückert von 1830-1832 [7], aber eine umfassende Ausführung scheiterte am Umfang des Unternehmens. Jahr rechnete 1848 vor, dass dazu wohl 48 Bände und pro Band mindestens ein Jahr Arbeit nötig wären: «so möchte ich wissen, welcher Einzelne je ein solches Werk würde vollenden, welcher Buchhändler es unternehmen, welcher Leser es anschaffen können!» [5; Band 1, S. VII]. Das Symptomen-Lexikon in der heutigen Form wurde in langjähriger Arbeit von Uwe Plate zusammengestellt und veröffentlicht. Es erschien zuerst 2004 in Buchform fünf dicke Bände mit insgesamt rund 8260 Seiten [8]. Die erste digitale Version erschien Anfang 2009, gegen Ende 2010 das erste Update 2.0 mit neuen Funktionen und Korrekturen, und im April 2011 folgte das Update 2.1.

Vereinfacht ausgedrückt ist das Symptomen-Lexikon eine Arzneimittellehre in multipler Anordnung. Die Symptome eines Mittels werden hier nicht mehr nur nach einem einzigen Kriterium sortiert und aufgeführt (herkömmlicherweise nach der Lokalisation). Im Symptomen-Lexikon sind drei weitere Kriterien ausgearbeitet, nämlich je einmal eine Sortierung nach Beschwerden/ Empfindung, nach Modalitäten und nach sogenannten «Zusatzzeichen». Lokalisation, Beschwerde und Modalität brauchen keine Erklärung. $\mathrm{Zu}$ den Zusatzzeichen gehören die Rubriken «Absatzweise» (z.B. Beschwerden, die kommen und gehen), "Anfallsweise» (anfallsartig, plötzlich Auftre-

\section{KARGER}

Fax +497614520714 Information@Karger.de www.karger.com
(๑) 2011 S. Karger GmbH, Freiburg
Beat Hanselmann

Culmannstrasse 23, 8006 Zürich, Schweiz

Tel. +41 443621710

www.beathanselmann.ch 
tendes), «Erstreckendes» (erstreckende Schmerzen, z.B. ein Reissen von der Hüfte zum Knie), «Halbseitige Beschwerden» (auf der linken oder rechten Seite auftretende Beschwerden), «Halbseitige Organe» (z.B. linker oder rechter Arm) und «Überlaufen» (z.B. überlaufende Hitze oder Kälte, Wallungen). Allgemein gilt, dass jedes Symptom aus Zeichen aufgebaut ist. Zeichen sind die Bausteine der Symptome. Und jedes Zeichen in einem Symptom kann als Kriterium zur Einsortierung verwendet werden. Das Phosphor-Symptom Stechen in den Knieen, Nachts, in Anfällen ist z.B. aus den Zeichen «Stechen» (eine Beschwerde), «Knie» (eine Lokalisation), «nachts» (eine Modalität) und «in Anfällen» (ein Zusatzzeichen) aufgebaut. In Hahnemanns «Chronischen Krankheiten» erscheint dieses Symptom einmal und ist als Nr. 1503 bei den Extremitäten mit anderen Kniesymptomen zwischen Ober- und Unterschenkel eingeordnet (Lokalisation im Kopf-zu-Fuss-Schema). Im Symptomen-Lexikon wird dieses Symptom in der Rubrik «Stechen» (im Teil «Beschwerden»), in der Rubrik «Nacht» (im Teil «Modalitäten»), in der Rubrik «Unterglieder - Knie» (im Teil «Organe») und in der Rubrik «Anfallsweise» (im Teil «Zusatzzeichen») aufgeführt. Es erscheint also insgesamt 4-mal! Oder das Symptom Wundschmerzendes Trockenheits-Gefühl im Schlunde, beim Schlucken, früh (Nr. 476) von Lycopodium taucht 5-mal auf, und zwar in den Rubriken: «Rachen/ Schlund» (Organe), «Trockenheit» und «Wundheit» (Beschwerden), «Morgen» und «Schlucken» (Modalitäten). Die Sortierung nach Organ (Lokalisation) ist übrigens erst seit dem Update von Ende 2010 im Symptomen-Lexikon vorhanden. Bei der Buchausgabe und der ersten digitalen Version war man darauf angewiesen, in den Arzneimittellehren oder Prüfungsberichten (die ja nach Lokalisation aufgebaut sind) nachzuschlagen.

Aus verschiedenen Gründen hat sich Uwe Plate im Symptomen-Lexi- kon auf 125 Arzneimittel beschränkt. Seine Quellen waren im Wesentlichen Hahnemanns «Reine Arzneimittellehre» [2] und «Chronische Krankheiten» [3] sowie die Arzneimittelprüfungen aus «Stapfs Archiv» [9] und die «Reine Arzneimittellehre» von Hartlaub und Trinks [10]. Plate hat also keine in Archiven verstaubten Handschriften ausgewertet und bisher unbekannte Informationen veröffentlicht, sondern «nur» die schon bekannten und gedruckten Prüfungssymptome neu angeordnet. Allerdings muss gleich hinzugefügt werden, dass man sich dieses «nur» in ganz grossen Anführungszeichen vorstellen muss, denn schliesslich handelt es sich um rund 76000 Symptome, von denen die meisten aus mehreren Zeichen zusammengesetzt sind und dadurch in verschiedene Rubriken einsortiert werden mussten.

Innerhalb der einzelnen Rubriken erscheinen die Arzneimittel alphabetisch. Kleine und kleinere Rubriken sind relativ übersichtlich, und man kann sich schnell einen Überblick verschaffen, welche Mittel hier in welcher Ausprägung vorkommen. Bei grossen Rubriken ist das nicht mehr möglich. Hier geben die Kollektaneen einen ersten, rein quantitativen Überblick. Sie gehören seit 2006 zum Symptomen-Lexikon. Der Begriff wird von Hahnemann in einem Brief an Bönninghausen verwendet: «Ihre Gewohnheit beim Lesen immer das Beste aufzuzeichnen, ist sehr nachahmungswerth, so mühevoll sie auch ist, und diese nur hat es möglich gemacht, daß Sie so große Kollektaneen haben zu Stande bringen können, worüber Jedermann erstaunen muß und selbst ich, der ich nicht unter die Unfleißigsten zu gehören mich bestrebte» [4; S. 44]. Er stammt aus dem Lateinischen (collectaneus = gesammelt) und bedeutet so viel wie "Lesefrüchte», d.h. eine Sammlung von Auszügen und Bemerkungen aus den Werken verschiedener Schriftsteller. Bei den Kollektaneen zum Symptomen-Lexikon handelt es sich um eine Tabelle, aus der ersichtlich wird, ob und wie oft ein bestimmtes Zeichen in einem Arzneimittel vorkommt. So sieht man z.B. schnell, dass Aurum, Oleander oder Rheum überhaupt keinen Bezug zu Blutungen, Fluor oder Hämorrhoiden besitzen. Auf der anderen Seite erkennt man, dass Atemnot am häufigsten bei Sulfur (39 Symptome), Sepia (35) und Phosphor (30), brennende Empfindungen und Schmerzen bei Sulfur (124), Kalium carbonicum (91) und Phosphor (82) sowie Weinen bei Lycopodium (14), Natrium muriaticum (14) und Platina (12) auftreten.

Ein solches Hilfsmittel wie das Symptomen-Lexikon ist zwar schön und gut, aber es stellt sich natürlich die Frage, inwiefern dieses Werkzeug denn nun mehr bietet als die bisher benutzten Arzneimittellehren und Repertorien und was man damit praktisch anfangen kann. Es sind vor allem zwei Bereiche, in denen es seine Stärken zeigt: beim Arzneimittelstudium und bei der Wahl des Mittels im Krankheitsfall.

\section{Arzneimittelstudium}

Beim Arzneimittelstudium möchte man das Wesen, die Idee oder die Essenz einer Arznei herausarbeiten; das, was immer wieder vorkommt, was typisch ist, was man sich merken will, damit man es beim Patienten wiedererkennt: der plötzliche Beginn bei Aconitum und Belladonna, die Wärmeverbesserung bei Arsenicum, die Verschlechterung zwischen 17 und 20 Uhr bei Lycopodium. Hahnemann spricht im Organon von dem Charakteristischen der Symptome nicht nur auf Seiten des Patienten, sondern auch bei der Arznei. Bönninghausen nannte es den Genius der Arznei, gebildet von den Zeichen, «welche, wie der rothe Faden in den Tauen der englischen Marine, durchgehends die ganze Reihe der Symptome jedes einzelnen Heilmittels durchlaufen" [11; Vorwort]. Und Jahr unterschied in seinen 
«Lehren und Grundsätzen» [12; \$103] zwischen «charakteristisch» und "wesentlich». Charakteristisch ist ein Zeichen dann, wenn es häufig und an (möglichst vielen) verschiedenen Organen vorkommt; wesentlich ist es, wenn es zwar häufig, aber nur an einer einzigen Lokalisation in Erscheinung tritt.

Constantin Hering hat in seiner Anleitung zum Arzneimittelstudium [13] vorgeschlagen, das ganze Mittel mindestens 4-mal unter verschiedenen Gesichtspunkten durchzulesen. Bei der Lektüre markiert man mit wechselnden Farben das erste Mal alle Organe, beim zweiten Mal Empfindungen und Befunde, beim dritten Mal Zeiten, Modalitäten und Seitenbeziehungen und schliesslich beim vierten Mal noch die «Verbindung der Zeichen». Am Ende dieser Arbeit kann man diejenigen Zeichen zusammenstellen, die zusammengehören (z.B. stechende Schmerzen oder eine Verschlimmerung durch Berührung), die aber über das ganze Arzneimittel verstreut sind. Genau diese Zusammenstellung ist im Symptomen-Lexikon bereits gemacht. Die Kollektaneen geben einen ersten Eindruck von den möglichen Stärken und Schwächen eines Mittels. Es ist z.B. auffällig, dass Coffea in der Prüfung kein einziges Symptom mit Herzklopfen hervorgebracht hat. Oder dass Nux vomica so viele Angstsymptome hat, dass es (mindestens von der numerischen Wahrscheinlichkeit her) mit Arsenicum, Calcium und Phosphor zu den grossen Angstmitteln gehören müsste. Wenn man nun diese Symptome im Lexikon nachschlägt, sieht man, dass Nux vomica tatsächlich einen starken Bezug zum Thema Angst hat, auch wenn es sich hauptsächlich durch Ängstlichkeit auszeichnet: Es hat in Hahnemanns «Reiner Arzneimittellehre» [2] 1301 Symptome. Davon gehören 55 zum Thema (oder Wortfeld) Angst. «Richtige» Angst erscheint 19-mal, davon 6-mal als «grösste», «äusserste», «höchste», «ausserordentliche» oder «unerträgliche» Angst. Seinen hohen Wert in den Kollektaneen erhält es durch 34 Symptome mit «Ängstlichkeit». Diese Ängstlichkeit wird nicht nur im Gemüt empfunden, sondern auch körperlich, z.B. Hie und $d a$ im Unterleibe, ängstlich drückende Blähungen (437) oder Aengstlichkeit in der Brust (700).

Man kann mit dem SymptomenLexikon leicht auch nur einen einzelnen Aspekt klären. Im «Therapeutischen Taschenbuch» [14] fehlt Phosphor in der Rubrik Verschlimmerung von Geräusch. Mit dem Symptomen-Lexikon kann man sich mit den Rubriken «Empfindlichkeit» (Beschwerden) und «Geräusch» (Modalitäten) schnell die relevanten Phosphor-Symptome zusammenstellen und sieht, dass es sich im «Taschenbuch» um einen der wenigen Fehler handeln muss: Ueberempfindlichkeit aller Sinne, besonders des Gehöres und Geruches (35); Mürrisch, jeder Gegenstand, besonders Menschen und Geräusch, sind ihm sehr zuwider (44); Grosse Schwäche im Kopfe, dass sie keinen Ton aufdem Klaviere vertragen konnte (110); Abends im Bette nicht schläfrig, und dann leiser Schlaf, so dass ihn jedes kleine Geräusch aufweckt (1720).

Oder die berühmte Durstlosigkeit von Pulsatilla. Im «Therapeutischen Taschenbuch» ist Pulsatilla bei Durst 2-wertig und bei Durstlosigkeit 4-wertig verzeichnet. Bei 1154 Prüfungssymptomen kommen mehr SymptomemitDurst(18)als mitDurstlosigkeit (10) vor. Wie ist das zu verstehen? Hahnemann gibt eine erste Erklärung in der Anmerkung zu Symptom Nr. 1039 [2; Band 2]: «Das Wechselfieber, was Pulsatille erregen kann, hat grösstentheils nur während der Hitze (nicht während des Frostes), seltner blos nach der Hitze oder vor dem Froste, Durst. Wo es bei blossem Hitzgefühle, ohne äusserlich bemerkbare Hitze, stehen bleibt, fehlt der Durst (...)». Dies sieht man durch die Symptome bestätigt. Durstlosigkeit erscheint 5-mal bei Frost/Schauder, 3-mal bei Hitzegefühl, aber auch 4-mal bei Hitze. Durst hingegen erscheint 6-mal bei Hitze, einmal nach Hitze und nie bei Frost. Der Durst von Pulsatilla kann stark sein (4-mal), und es besteht ein deutliches Verlangen nach Bier (6 Symptome). Aufgrund der Prüfung kann für Pulsatilla also nicht generell eine Durstlosigkeit - und Durst als Gegenindikation postuliert werden.

\section{Mittelwahl im Krankheitsfall}

Hahnemann lehrt uns im berühmten $\$ 153$ des Organon [1], dass wir «die auffallendern, sonderlichen, ungewöhnlichen und eigenheitlichen (charakteristischen) Zeichen und Symptome des Krankheitsfalles, besonders und fast einzig fest in's Auge zu fassen (haben); denn vorzüglich diesen, müssen sehr ähnliche, in der Symptomenreihe der gesuchten Arznei entsprechen, wenn sie die passendste zur Heilung sein soll». Wir suchen also ein «sehr» (und nicht nur ein irgendwie) ähnliches Heilmittel; wir suchen die "passendste» Arznei. Und wir suchen in den Symptomenreihen, also in den Arzneimittelprüfungen. Diese sind das Sicherste und am wenigsten Bearbeitete oder Interpretierte, auf das wir unsere Arbeit stützen können. Es mag zwar ungewohnt oder gar seltsam erscheinen, einen Krankheitsfall direkt und ausschliesslich mit der reinen Arzneimittellehre lösen $\mathrm{zu}$ wollen, aber alle zusätzlichen Informationen zu einem Arzneimittel sind mindestens eine Stufe weniger verlässlich als die reinen Prüfungssymptome - seien dies nun Heilwirkungen oder klinische Erfahrungen, Miasmazugehörigkeit oder zentrale Wahnidee.

Natürlich kann hier nicht im Detail beschrieben werden, wie ein Arzneimittel mithilfe des Symptomen-Lexikons gewählt werden kann. Uwe Plate hat das in den Einführungen zum Symptomen-Lexikon und in seinem Buch «Hahnemanns Arbeitsweise» 
[15] beschrieben, und mittlerweile gibt es im deutschsprachigen Raum auch verschiedene Seminare, auf denen man sich dieses Wissen aneignen kann.

Praktisch geht man am besten so vor, dass man zuerst mithilfe der Kollektaneen oder eines Repertoriums eine Vorauswahl trifft. Manchmal kann man auch mit einer guten Kombination von Zeichen beginnen (siehe Beispiele 1 und 2). Wenn man sauber gearbeitet hat, erhält man einen (mehr oder weniger grossen) Mittelpool, in dem das für den vorliegenden Fall angezeigte Mittel mit grosser Wahrscheinlichkeit enthalten sein muss. Als Repertorium hat sich das «Therapeutische Taschenbuch» [14] sehr bewährt. Die modernen grossen Repertorien sind für diese Arbeit nicht sonderlich geeignet.

Die Mittel im Pool werden nun weiter differenziert. Dabei beginnt man mit den wichtigsten, in der Hierarchie am höchsten stehenden Beschwerden und geht dann schrittweise von den Haupt- bis zu den $\mathrm{Ne}$ bensymptomen, von den beschwerlichsten bis $\mathrm{zu}$ den harmlosesten Zeichen. In einer Art "Arzneimittelcasting» müssen die Mittel also beweisen, dass sie den im Krankheitsfall vorkommenden Symptomen und Zeichen auch tatsächlich und vor allem im Detail gerecht werden. Ist dies nicht der Fall, entfallen sie. Man untersucht die Arzneien und vergleicht sie mit dem Krankheitsfall so lange, bis nur noch ein einziges Mittel übrig bleibt. Dieses ist nun das «passendste zur Heilung» (oder im schlechtesten Fall das am wenigsten unpassende). Als besonders aussagekräftig haben sich Zeichenkombinationen erwiesen, also z.B. Schwindel mit Übelkeit oder Durchfall nach dem Essen. Eine Arznei, die eine solche Kombination von zwei (oder mehr) Zeichen bereits in der Prüfung hervorgebracht hat, ist einer anderen Arznei deutlich überlegen, die diese Zeichen zwar auch, aber ohne gleichzeitigen oder inneren Zusammen- hang erzeugt hat. An vier einfachen Beispielen soll das gezeigt werden.

\section{Beispiel 1}

Die oben erwähnte Zeichenkombination Durchfall nach dem Essen: Es gibt viele Mittel mit einer allgemeinen Verschlimmerung nach Essen. Durchfall nach Essen haben in der Prüfung allerdings nur 9 Arzneien erzeugt, 8 davon mit jeweils nur einem einzigen Symptom. Aber bei Borax finden sich folgende vier Symptome: Nach dem Mittagessen, Kollern im Leibe und Durchfall (194); Gleich nach Tische, Durchfall, mit Schwäche in den Gelenken und Beinen, was nach dem Gehen sich bessert (195); Bald nach dem Frühstücke, Schneiden im rechten Hypochonder, quer durch den Bauch nach abwärts, darauf Durchfall, und zwar plötzliches Entleeren auf einmal (196); Nach dem Frühstücke, Durchfall, $4 \mathrm{Mal}$ nach einander (197). Das bedeutet, dass Borax in diesem Punkt alle anderen Mittel deutlich in den Schatten stellt und - wenn es sich auch sonst eignet und keine Gegenanzeigen vorliegen - in einem Fall gegeben werden sollte, bei dem Durchfall nach dem Essen das Hauptproblem ist.

\section{Beispiel 2}

In einem Fall bestand die Hauptbeschwerde in kneifenden Schmerzen in der Nabelgegend, schlimmer nach dem Essen und schlimmer durch Druck. Bei der Kombination «Essen» (Modalitäten) und «Kneifen» (Beschwerden) finden sich insgesamt 40 Mittel, die meisten davon allerdings mit nur einem Treffer. Von den 13 Mitteln mit 2 oder mehr Treffern hat einzig Ranunculus bulbosus auch noch eine Verschlimmerung von kneifenden Schmerzen durch Druck. Die übrigen Symptome passten ebenfalls zu diesem «kleinen» Mittel, das dann auch schnell und zuverlässig heilte.

\section{Beispiel 3}

Ein 14 Monate altes Mädchen reagierte auf eine Impfung mit längerem
Schreien und Jammern in der Nacht. Weitere Symptome waren nicht vorhanden. Welches der bekannten Impffolgemittel sollte man da geben? Im digitalen Symptomen-Lexikon finden sich 31 Mittel bei der Kombination «Nacht» (Modalitäten) und «Klagen» (Beschwerden), darunter Silicea mit den meisten Treffern und drei guten Symptomen: Er träumt Nachts viel und schreit im Schlafe auf (1115); Der Knabe ist Nachts unruhig und schreit (1116); Der Knabe erwacht Nachts mit heftigem Weinen, kömmt nicht zum Besinnen, sondern jammert ängstlich mit Lallen (1117). Eine Dosis Silicea C30 zeigte in der ersten Nacht noch keine grosse Wirkung, aber am nächsten Tag war das Mädchen ruhiger und ab der zweiten Nacht schrie es nicht mehr.

\section{Beispiel 4}

Ein 43-jähriger Mann schreckt seit 3-4 Monaten in der Nacht mit relativ intensivem Herzklopfen auf. Der Puls ist nicht erhöht; er verspürt eine Art Vibrieren in der Herzregion. Das ist so störend, dass er nicht mehr einschlafen kann. Manchmal dauert diese Empfindung nur ein paar Stunden, manchmal auch bis in den nächsten Tag hinein. Im Liegen schlimmer, nach dem Aufstehen nicht mehr merkbar. Am nächsten Tag kann er im Bereich der Nase ein Gefühl von Kribbeln (wie von Meerrettich) oder von Fieber haben. Alkohol kann diese Erscheinungen auslösen. In den seltenen Fällen, in denen diese Erscheinung länger andauert, spürt er ein Kribbeln bis in die Arme und Beine, im Sitzen schlimmer, die linke Seite etwas mehr betroffen. Untersuchungen beim Hausarzt ohne Befund. Er steht vor einem Arbeitsplatzwechsel. Er hat über 18 Monate gesucht und fühlt sich nun psychisch «erlöst». Die Repertorisation mit dem «Therapeutischen Taschenbuch» [14] ergibt einen Mittelpool mit 14 Mitteln (Abb. 1). Wie die weitere Auswertung mit dem Symptomen-Lexikon zeigt, haben nur 7 davon überhaupt Herz- 
Abb. 1.

Repertorisation mit dem «Therapeutischen Taschenbuch» [14] und weitere Differenzierung mit dem SymptomenLexikon.

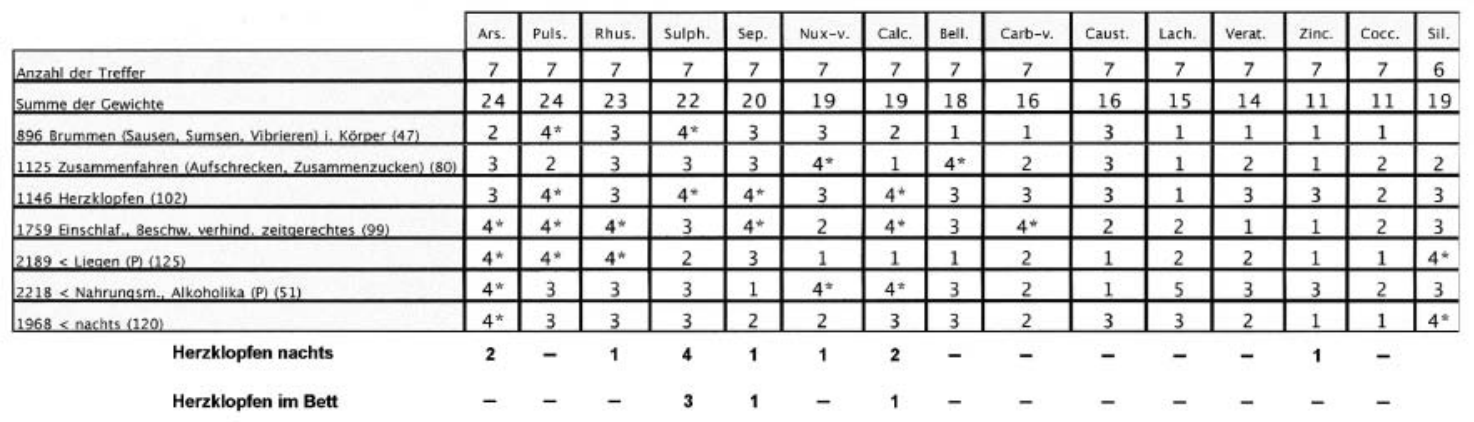

klopfen in der Nacht oder im Bett. Von diesen führt Sulfur deutlich. Eine Gabe Sulfur C30 sorgte insofern für eine Besserung, dass der Mann manchmal noch beim Aufwachen kurz Herzklopfen empfand, das sich aber innerhalb von 5-10 min wieder beruhigte. Keine Beschwerden lagen mehr bei Alkohol vor. Nach 13 Monaten, als es auf der Arbeit viel Stress gab, zeigten sich die Symptome nochmals in leichter Form und verschwanden schnell wieder mit Sulfur.

\section{Fazit}

In der Fachpresse wurde ein zum Teil heftiger Streit für und wider das Symptomen-Lexikon ausgetragen. Und wie so oft in der Homöopathie wusste jede Partei die Wahrheit und das Alleinseligmachende natürlich auf ihrer Seite. Dabei sollte man nicht vergessen, dass man die freie Wahl hat. Man kann ein Werkzeug und Hilfsmittel gebrauchen und heraus- finden, welchen Nutzen es einem bringt und ob es einen bei der Arbeit unterstützt.

\section{Literatur}

1 Schmidt JF (Hrsg): Samuel Hahnemann. Organon der Heilkunst. Standardausgabe der 6. Auflage. Neuausgabe 1999. Auf der Grundlage der 1992 vom Herausgeber bearbeiteten textkritischen Ausgabe des Manuskripts Hahnemanns (1842). Heidelberg, Haug, 1999.

2 Hahnemann S: Reine Arzneimittellehre. Dresden/Leipzig, Arnold, 1825-1833.

3 Hahnemann S: Die chronischen Krankheiten, ihre eigenthümliche Natur und homöopathische Heilung. Antipsorische Arzneien. Zweite, viel vermehrte und verbesserte Auflage. Dresden/Leipzig/Düsseldorf, Arnold/Schaub, 1835-1839.

4 Stahl M: Der Briefwechsel zwischen Samuel Hahnemann und Clemens von Bönninghausen. Heidelberg, Haug, 1997.

5 Jahr GHG: Ausführlicher Symptomen-Kodex der Homöopathischen Arzneimittellehre. Zweiter Theil. Systematisch-alphabetisches Repertorium. Leipzig, Bethmann, 1848.

6 Weber GA: Systematische Darstellung der antipsorischen Arzneimittel in ihren reinen Wirkungen: nach Dr. S. Hahnemanns Werke: Ueber die chronischen Krankheiten, ihre eigenthümliche Natur und homöopathische Heilung. Braunschweig, 1830
7 Rückert ETF: Systematische Darstellung aller bis jetzt gekannten homöopathischen Arzneien, mit Inbegriff der antipsorischen, in ihren reinen Wirkungen auf den gesunden menschlichen Körper. Leipzig, Schumann, 1830-1832.

8 Plate U: Symptomen-Lexikon der Materia Medica, nach einer Idee des Begründers der Homöopathie Samuel Hahnemann. Braunschweig, 2004. www.symptomenlexikon.de.

9 Gypser K-H, Waldecker A, Wilbrand R (Hrsg): Gesammelte Arzneiprüfungen aus Stapfs «Archiv für die homöopathische Heilkunst» (1822-1848). Heidelberg, Haug, 1991-1994.

10 Hartlaub CGC, Trinks CF: Reine Arzneimittellehre. Leipzig, Brockhaus, 1828-1831.

11 von Bönninghausen CMF: Die Körperseiten und Verwandtschaften. Münster, Regensberg, 1853.

12 Jahr GHG: Die Lehren und Grundsätze der gesamten theoretischen und praktischen Homöopathischen Heilkunst. Eine apologetisch-kritische Besprechung der Lehren Hahnemanns und seiner Schule. Stuttgart, Liesching, 1857.

13 Hering C: Ueber das Studium der homöopathischen Arzneimittellehre; in Wirkungen des Schlangengiftes, zum ärztlichen Gebrauch vergleichend zusammengestellt. Allentaun/Leipzig, Blumer, 1837.

14 Gypser K-H (Hrsg): Bönninghausens Therapeutisches Taschenbuch. Stuttgart, Sonntag, 2002.

15 Plate U: Hahnemanns Arbeitsweise mit dem Symptomen-Lexikon ... dargestellt an Praxisfällen aus seinen Krankenjournalen. Braunschweig, Selbstverlag, 2003. 